\title{
TAMING A SURFACE BY PIERCING WITH DISKS
}

\author{
W. T. EATON ${ }^{1}$
}

In [3] and [2], respectively, Bing proves the following two theorems.

THEOREM 1. A 2-sphere $S$ in $E^{3}$ is tame from complementary domain $U$ if and only if $U$ is 1-ULC.

THEOREM 2. If $S$ is a 2-sphere in $E^{3}$ and $U$ is a complementary domain of $S$ then there exists a 0-dimensional $F_{\sigma}$ set $F \subset S$ such that $U \cup F$ is 1-ULC. Furthermore if $\left\{X_{i}\right\}$ is a sequence of sets in $S$, each of which is either a tame finite graph or a tame Sierpinski curve, then $F$ may be chosen in $S-U X_{i}$.

The above two theorems suggest a procedure for showing that a given condition restricting the embedding of $S$ implies $S$ is tame from $U$. Namely, it may be possible to use the condition to slightly adjust a map $f$ from a disk $D$ into $U \cup F$ so that the new image of $D$ lies entirely in $U$ while $f \mid \operatorname{Bd} D$ is unaltered. The facts that $f(D) \cap F$ is compact 0 -dimensional and $F$ lies in $S-U X_{i}$ may also be helpful while adjusting $f$.

The above technique is employed in this paper to answer in the affirmative the following question asked in [1] and [5]. Is a 2-sphere in $E^{3}$ tame if it can be pierced at each arc with a tame disk? Other illustrations of this procedure may be found in [7] and [8].

Definition. A disk $D$ is said to pierce sphere $S$ at arc $A \subset S$ if Bd $A \subset \mathrm{Bd} D$, Int $A \subset$ Int $D$ and the two components of $D-A$ lie in different complementary domains of $S$.

Definition. If $J$ is a simple closed curve in 2 -sphere $S$ and $U$ is a complementary domain of $S$ then $J$ can be collared from $U$ by $A$ if $A$ is an annulus such that $J \subset \mathrm{Bd} A$ and $A-J \subset U$.

The reader is referred to $[2]$ and [3] for definitions of other terms used in this paper.

THEOREM 3. If $S$ is a 2-sphere in $E^{3}$ then $S$ is tame if and only if each simple closed curve in $S$ can be collared from each complementary domain of $S$ by a tame annulus.

Presented to the Society, April 20, 1968 under the title Tameness of certain types of spheres; received by the editors November 21, 1968.

1 Work on this paper supported in part by NSF Grant GP-8888. 
Proof. Let $U$ be a complementary domain of $S$. We will show that $U$ is $1-U L C$ and apply Theorem 1 to conclude that $S$ is tame from $U$. Suppose $\epsilon>0$, then it follows from Theorem 2 that there exists a $\delta>0$ such that if $f$ is a map of the boundary of a disk $D$ into a $\delta$-subset in $U$ then $f$ may be extended to $D$ so that

(1) $f(D)$ is an $\epsilon / 2$ subset of $\bar{U}$,

(2) $f(D) \cap S$ is 0 -dimensional, and

(3) $f(D) \cap S \subset$ Int $A$ where $A$ is an $\epsilon / 2$-annulus in $S$.

It follows from (2) that there exists a homeomorphism $h$ of $\left\{(x, y) \mid 1 \leqq x^{2}+y^{2} \leqq 4\right\}$ onto $A$ such that the arc

$$
B=h(\{(x, y) \mid 1 \leqq x \leqq 2, y=0\})
$$

misses $f(D)$. From the hypothesis there exists a disk $C$ that pierces $S$ at $B$ and also misses $f(D)$. It follows from (1) and (3) that there exists an open set $N$ such that $N \cap S=\operatorname{Int} A, N \cap(f(\operatorname{Bd} D) \cup \operatorname{Bd} C)=\phi$, and Diam $f(D) \cup N<\epsilon$. The proof will be completed by adjusting the singular disk $f(D)$ in $N$ so that the new image of $D$ lies in $U$.

It follows from the hypothesis that for each $t \in(1,2)$ there exists a tame annulus $A_{t}$ such that

(4) $A_{t} \cap S$ is the simple closed curve $J_{t}=h\left(\left\{(x, y) \mid x^{2}+y^{2}=t^{2}\right\}\right)$, and

(5) $A_{t} \subset N \cap \bar{U}$.

We need the following well-known lemma from general topology. An easy proof may be obtained by showing that the space of all continuous functions from an element of $\mathfrak{C}$ into $X$ is a separable metric space under the sup-norm metric. The elements of $\mathfrak{C}$ may then be considered as points in this separable metric space.

LEMma 4. If $\mathfrak{e}$ is an uncountable collection of homeomorphic compact subsets of a separable metric space $X$ then there exists a countable subcollection $\mathfrak{C}^{\prime}$ of $\mathfrak{C}$ such that if $Y \in \mathrm{C}-\mathfrak{C}^{\prime}$ then there exists a sequence $\left\{Y_{i}\right\} \subset \mathrm{C}-\mathfrak{e}^{\prime}$ which converges homeomorphically to $Y$.

Apply Lemma 4 by letting $\mathcal{C}=\left\{A_{t} \mid t \in(1,2)\right\}$ and consider an $A_{t} \in \mathfrak{C}-\mathfrak{C}^{\prime}$. There is a sequence $\left\{A_{t_{i}}\right\} \subset \mathfrak{e}-\mathfrak{e}^{\prime}$ converging homeomorphically to $A_{t}$; consequently, there exists an integer $i$ such that $A_{t}$ and $A_{t_{i}}$ are homeomorphically so close that there exists a singular annulus $B_{t}$ (resulting from a homotopy) such that

(6) $\mathrm{Bd} B_{t}=\left(\operatorname{Bd} A_{t} \cup \mathrm{Bd} A_{t_{i}}\right)-S$, and

(7) $B_{t} \subset N \cap U$.

The union of $A_{t}, B_{t}$ and $A_{t_{i}}$ is a singular annulus which has no singularities near its boundary. Dehn's lemma [9] is applied to replace $A_{t} \cup B_{t} \cup A_{t_{i}}$ with a nonsingular annulus $C_{t}$ with the same boundary. 
Hence there exists a countable set $P \subset(1,2)$ such that if $t \in(1,2)-P$ there exists a tame annulus $C_{t}$ such that

(8) $\mathrm{Bd} C_{t}=J_{t} \cup J_{s}$ for some $s \in(1,2)$, and

(9) Int $C_{t} \subset N \cap U$.

Let $D_{t}$ be the annulus lying in $A$ with the same boundary as $C_{t}$. It is straightforward to show that there exists a countable set $Q \subset(1,2)$ such that $s \in Q$ whenever

$$
J_{s} \nsubseteq \cup \underset{t \in(1,2)-P}{\operatorname{Int}} D_{t}
$$

Furthermore there exists a countable set $R \subset(1,2)-P$ such that

$$
\text { U } \operatorname{Int}_{t \in(1,2)-P} D_{t}=\bigcup_{t \in R} \text { Int } D_{t}
$$

The set

$$
Y=\left(\bigcup_{s \in Q} J_{s}\right) \cup\left(\bigcup_{t \in R} \operatorname{Bd} D_{t}\right)
$$

is the union of a countable number of tame simple closed curves, so by Theorem 2 there exists a 0 -dimensional set $F \subset S-Y$ such that $U \cup F$ is 1 -ULC.

It follows that there exists an open set $V$ containing $f(D) \cap S$ such that loops in $V \cap U$ can be shrunk to points in $(U \cup F) \cap(N-C)$. It is straightforward to find a finite collection $E_{1}, \cdots, E_{k}$ of disjoint disks in $D$ such that $f^{-1}(S) \subset \bigcup_{i=1}^{k} E_{i} \subset$ Int $D$ and $f\left(\operatorname{Bd} E_{i}\right) \subset V \cap U$. The map $f \mid \operatorname{Bd} E_{i}$ is extended to a map $f_{i}: E_{i} \rightarrow(U \cup F) \cap(N-C)$. The maps $f_{i}(i=1, \cdots, k)$ and $f \mid D-\bigcup_{i=1}^{k} E_{i}$ are pieced together to form a mapg: $D \rightarrow U \cup F$. Note that $g(D) \cap S \subset F \cap \operatorname{Int} A \subset\left(\cup_{t \in R}\right.$ Int $\left.D_{t}\right)-Y$.

There exists a finite set $T \subset R$ such that $g(D) \cap S \subset$ Int $_{t \in T} D_{t}$. We assume that $C_{t}$ and $C_{t^{\prime}}$ are in general position whenever $t, t^{\prime} \in R$ and $t \neq t^{\prime}$. Note that for each $s \in T$ a simple closed curve $K$ in Int $C_{8}$ links Bd $C$ if and only if $K$ separates the boundary components of $C_{s}$ in $C_{s}$; thus, a simple closed curve in Int $C_{t} \cap \operatorname{Int} C_{t^{\prime}}$ bounds a disk in Int $C_{t}$ if and only if it bounds a disk in Int $C_{t^{\prime}}$. Using standard disk and annulus trading techniques we alter the collection $\left\{C_{t}\right\}_{t \in \boldsymbol{T}}$ to form a new finite collection $\left\{F_{i}\right\}_{i=1}^{n}$ of annuli such that

(10) Bd $F_{i}=J_{s} \cup J_{t}$ for some $s, t \in(1,2)$,

(11) Int $F_{i} \subset N \cap U$,

(12) Int $F_{i} \cap$ Int $F_{j}=\phi$ whenever $i \neq j$, and if $G_{i}$ is the annulus lying in $A$ with the same boundary as $F_{i}$ then

(13) $g(D) \cap S \subset \bigcup_{i=1}^{n}$ Int $G_{i}$.

For $i=1, \cdots, n$ each component of the boundary of $F_{i}$ links Bd $C$ and $F_{i} \cap \mathrm{Bd} C=\phi$; consequently, each simple closed curve in (Int $F_{i}$ ) 
$-C$ bounds a disk in Int $F_{i}$. Since $g(D) \cap C=\phi$, it follows that there exists a finite collection $D_{i j}$ of disjoint disks in Int $F_{i}$ such that $g(D) \cap F_{i} \subset \cup_{j} D_{i j}$. The union, $\cup_{i, j} D_{i j}$, separates $g(\mathrm{Bd} D)$ from $g(D) \cap S$ on $g(D)$ so it follows from the Tietze Extension Theorem as indicated in $\left[4\right.$, Lemma 6] that there is a map $f^{\prime}: D \rightarrow(U \cap N)$ $\cup(f(D)-N)$ such that $f^{\prime}|\operatorname{Bd} D=g| \operatorname{Bd} D=f \mid \operatorname{Bd} D$. Since Diam $f(D)$ $\cup N<\epsilon$, Diam $f^{\prime}(D)<\epsilon$ and we have $U$ is 1-ULC. An application of Theorem 1 completes the proof.

THEOREM 5. A 2-sphere $S$ in $E^{3}$ is tame if and only if $S$ can be pierced at each arc with a tame disk.

Proof. The lemma after Theorem 4 of [6] shows that the hypothesis of Theorem 3 is satisfied.

R. J. Daverman has recently weakened the hypothesis of Theorem 5 to include the case where $S$ can be pierced at each arc with a singular disk and each arc of $S$ is tame.

\section{REFERENCES}

1. R. H. Bing, Embedding surfaces in 3-manifolds, Proc. Internat. Congr. of Mathematicians, 1962, pp. 457-458.

2. - - Pushing a 2-sphere into its complement, Michigan Math. J. 11 (1964), $33-45$.

3. - A surface is tame if its complement is 1-ULC, Trans. Amer. Math. Soc. 101 (1961), 294-305.

4. C. E. Burgess, Characterizations of tame surfaces in $E^{3}$, Trans. Amer. Math. Soc. 114 (1965), 80-97.

5. - Surfaces in $E^{3}$, Topology Seminar, Wisconsin, Princeton Univ. Press, Princeton, N. J., 1966, pp. 73-82.

6. - Criteria for a 2-sphere in $S^{3}$ to be tame modulo two points, Michigan Math. J. 14 (1967), 321-330.

7. W. T. Eaton, A note about locally spherical spheres, Canad. J. Math. (to appear). 378.

8. - Cross sectionally simple spheres, Bull. Amer. Math. Soc. 75 (1969), 375-

9. C. D. Papakyriakopoulos, On Dehn's lemma and the asphericity of knots, Ann. of Math. 66 (1957), 1-26.

UNIVERSITY OF TENNESSEE 